\title{
Micro Digital Relay Protection Device Based on Advance Multicore Processor
}

\author{
Dehui Zeng ${ }^{1,}$, , Weitao Yang ${ }^{2, b}$ \\ ${ }^{1}$ School of Electric Power, South China University of Technology, \\ Guangzhou, 510640, China \\ 2 School of Electric Power, South China University of Technology, \\ Guangzhou, 510640, China \\ aemail: danielzdh@126.com, bemail: 451031804@qq.com
}

Keywords: Relay Protection; Multicore Processor; Digital Substation; IEC61850

\begin{abstract}
The hardware and software design scheme of the single board micro digital relay protection device is introduced in this paper. The hardware platform of the device is based on AM5728 embedded multicore processor of TI (Texas Instruments) Company which has ARM cores and DSP cores. The software platform of the device is based on TI-RTOS real-time operating system. Well-designed hardware and software let the device has advantages in high reliability, powerful computational capability, low cost and small size, which meet the general requirements of protection and monitor devices for digital substation.
\end{abstract}

\section{Introduction}

With the development of intelligent electrical equipment, especially the emergence of intelligent switch, electronic instrument transformer and the high speed Ethernet technology, substation automation technology has entered a new stage of digitization. Cable connections between primary equipment such as traditional instrument transformers, switches and secondary equipment have been canceled. In digital substation electronic instrument transformers, intelligent primary equipment and merging units are all connected by optical fiber ${ }^{[1,2]}$.

With the application of IEC61850 protocol and the popularity of digital substation technology, the new digital protection based on IEC61850 has been widely used ${ }^{[3,4]}$. In order to meet the needs of network communication and data processing, multiple processor circuit boards have been used in the existing digital protection devices ${ }^{[5-7]}$. But such a structure has disadvantages of large volume; high cost; complex operation and so on. These disadvantages have become the obstacles to the promotion of digital protection in distribution network.

The purpose of this paper is to try to design a single-board micro digital relay protection platform based on the existing digital relay protection. Traditional plug-in design is abandoned on the device, and all functions are integrated into a circuit board, madding the device have advantages in high reliability, low cost and small occupied area.

\section{Overall Structure of The device}

Since the seventies of the 20th century, the platforms of microcomputer relay protection upgrade constantly, but most of them are designed with one or more processors, and sampling module, communication module, digital I/O module, power supply module and a man-machine interactive module around the processor. At present, there are many kinds of processors used in relay protection, such as DSP+ARM ${ }^{[8]}$; DSP+ FPGA ${ }^{[9-10]}$; Multi-DSP ${ }^{[11-12]}$ and so on. DSP+ARM architecture is most widely used.

This device uses the AM5728 processor of TI (Texas Instruments) Company. AM5728 is ARM based SOC with dual-core ARM Cortex-A15 RISC CPUs, and two TI-C66x floating-point DSP cores. Besides the display subsystem and coprocessors include dual-core ARM Cortex-M4 RISC CPUs and 
two PRU (programmable Real-Time Unit) kernels are provide in this processor.

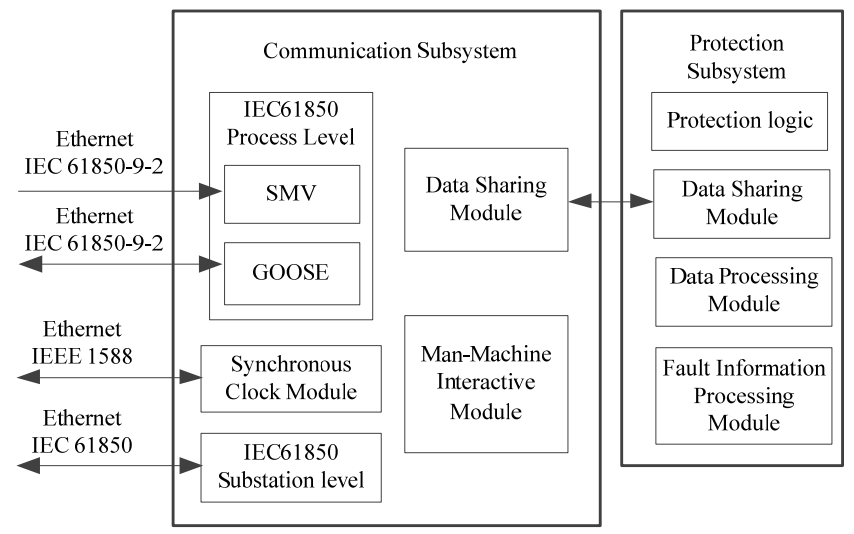

Fig.1. Structure of the micro digital relay protection

The structure of the micro digital relay protection platform is shown in Fig. 1. This platform can be divided into two parts, which are communication subsystem and protection subsystem. Communication subsystem is mainly composed of two Cortex-A15 ARM kernels and RPU kernels of AM5728. As Fig. 2 the digital protection is in bay level, so IEC 61850 Process level communication module of communication subsystem is responsible for receiving and analyzing the SV packages sent by the merging unit and GOOSE packages sent by intelligent primary equipment. The substation layer communication module is used to exchange information with substation-backgrounds-supervising system. The exchange service contains many contents, which include setting upload and download, protection information upload, remote control command receiving, fault-record file upload. The synchronous clock module realizes the synchronization of the sampling data in the substation according to the IEC1588_V2 precision time protocol. The data sharing module is designed for information interaction with the protection subsystem.

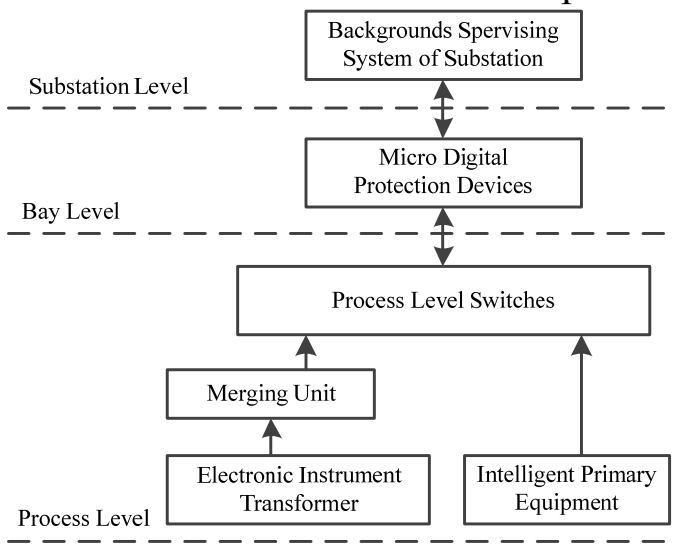

Fig.2 Position of digital position protection in digital substation

The protection function subsystem is composed of C66x DSP AM5728 kernel, which is divided into data sharing module; data processing module; protection logic module and fault information processing module. The data sharing module is used to exchange data exchange with the communication subsystem. The data processing module is applied for calculating the effective value of the electric quantity and extracting the positive or negative sequence component. The protection logic module is designed for the realization of various protection principles. The fault information processing module is responsible for recording the action report information and generating the recording data.

\section{Hardware Design}

The hardware of the device is composed of a processor module and a device motherboard. And the processor module is connected to the motherboard through two board to board connectors. Such a structure has several advantages: Firstly, in order to improve reliability, data processing and communication interfaces are separated; Secondly, high density and low density PCBs are separated 
to reduce the cost; Thirdly, The protection function can be enhanced by changing the processor module.

\section{Processor Module}

Processor module is the core to realize the function of communication and protection. It is based on AM5728 processor and components for supporting AM5728 such as large-capacity DRAM and Flash memory; power supply chips and digital clock chips. The hardware structure of the processor module is shown in Fig.3.

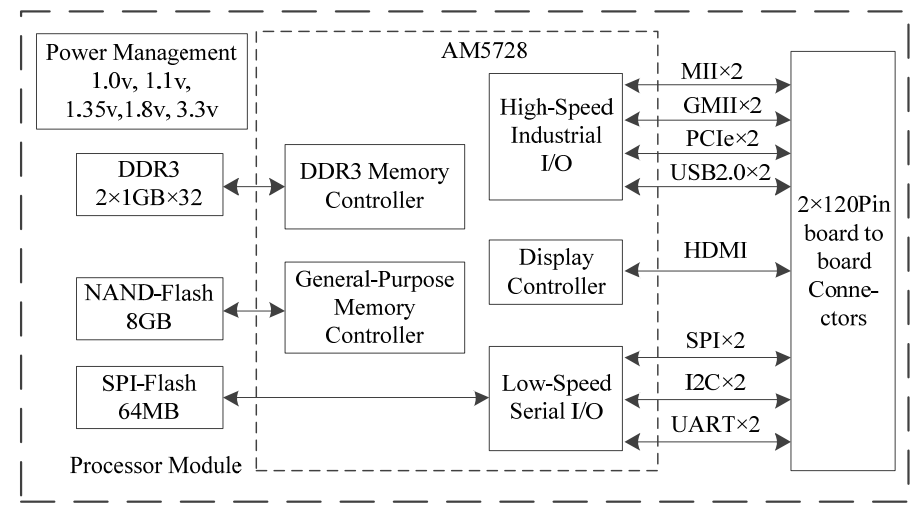

Fig.3 Schematic diagram of Processor module

In power management part, a high efficiency synchronous buck chip (TPS659037) is adopted to provide multi-power supply for AM5728 and other chips in Processor module. K4E8E324EB is used as LPDDR3 memory which has 32-bit bus width and 1GB capacity. KLM8G1GEN is used as Flash memory which has the capacity of 8GB. In processor module, most of the I/O interfaces of AM5728 are drawn to board-to-board connectors. The Gigabit Medium Independent Interface (GMII) and the Medium Independent Interface (MII) can provide two independent Gigabit Ethernet interfaces which support IEEE1588 precise time synchronization protocol and two Fast Ethernet interfaces. USB interfaces can connect mouse, keyboard and U disk. HDMI interface is used to connect monitoring display, and the UART interface can provide RS232 and RS485 serial communication ports.

AM5728 runs in high frequency and needs multi-power supply, so in order to increase the reliability and the signal integrity, we design the processor module with ten layers, including four power layers and six signal layers. Besides, isometric routing technique is used in high speed parallel buses to minimize EMI coupling. As an example, the data bus and address bus of DDR3 are matched within 50 mils; the data bus of NAND flash are matched within 100 mils and the data bus of GMII interface are matched within 50 mils. What's more, high-speed serial buses such as USB interfaces are routed as differential pairs.

\section{Motherboard}

Motherboard is designed around the processor module with power supply part, Ethernet communication part, serial interface part, display part and battery part. The hardware structure of the Motherboard is shown in Fig.4.

AM5728 has built-in Ethernet switching module which has integrated MAC layer functions with two GMII interfaces and two MII interfaces, so we use Ethernet physical layer transceivers to extend several Ethernet Connectors. Then we select 88E1116R as Gigabit Ethernet physical layer transceivers and AFCT-5701Z as Gigabit SFP Fiber Optic Connector. For fast Ethernet, we select KSZ8721CL as physical layer transceivers and HFBR-57E0LZ as SFP Fiber Optic Connector. What's more, two Gigabit Ethernet connectors are independent and IEEE1588 version 2 time stamping is supported on both of them. 


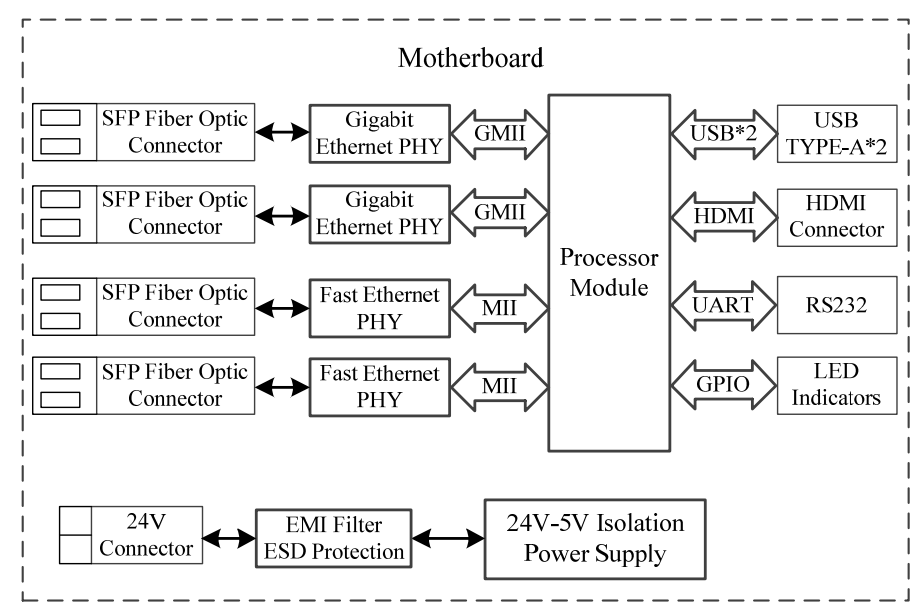

Fig.4 Schematic diagram of Motherboard

An isolated DC-DC module is used as power supply for the whole device. The input of this DC-DC module is $24 \mathrm{~V}$ and the output is $5 \mathrm{~V} / 8 \mathrm{~A}$. Besides, EMI filter and ESD protection circuit are added on the motherboard to improve the reliability of power supply.

The USB ports and HDMI port on the motherboard are used to achieve man-machine interaction. The RS232 serial port generated by UART is used to debugging. Four LED indicators on the motherboard are used to indicate the device states including running, alarm, protection action and device abnormal.

\section{Software Design}

The software of micro digital protection is developed based on TI-RTOS which is an open-source real-time operating system. TI-RTOS fully supports TI's multicore ARM and DSP including AM5728. The TI-RTOS Kernel provides a uniform set of the operating system APIs (application programming interfaces) across all cores. TI-RTOS Kernel also offers shared image and SMP (Symmetric Multi-Processing) supports for appropriate devices. The associated IPC (Inter-Process Communication) Package provides an extensive set of multicore communication mechanisms that simplify development of distributed applications and leverage any hardware mutual exclusion features for maximum performance.

The software is divided into three parts: communication program, protection program and monitoring program. The communication program and monitoring program mainly run in ARM cores of AM5728 and protection program runs in DSP cores. Data of these programs are shared by Share-RAM. The main functions of these programs and their relationship are shown in Fig.5.

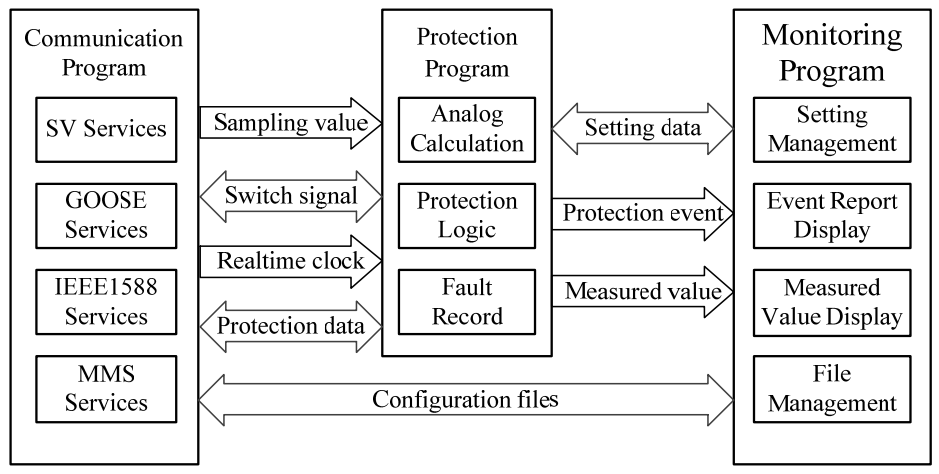

Fig.5 Overall structure of software

Information including sampling values, switch signals and real-time clock will be obtained by communication program from the process layer network. Protection data comprising setting data; configuration data and remote data will be given by communication program from substation layer network. All of this information is shared to the protection program. Monitoring program is used to realize the man-machine interactive function and the protection device debugging. 


\section{Protection program}

Since protection program is the most important part of the software, protection program runs in a timer-interrupt service routine. The interrupt interval is 500us and a synchronous clock signal generated by IEEE1588 service is used to fix the interrupt interval. The running process of the protection program is shown in Fig.6.

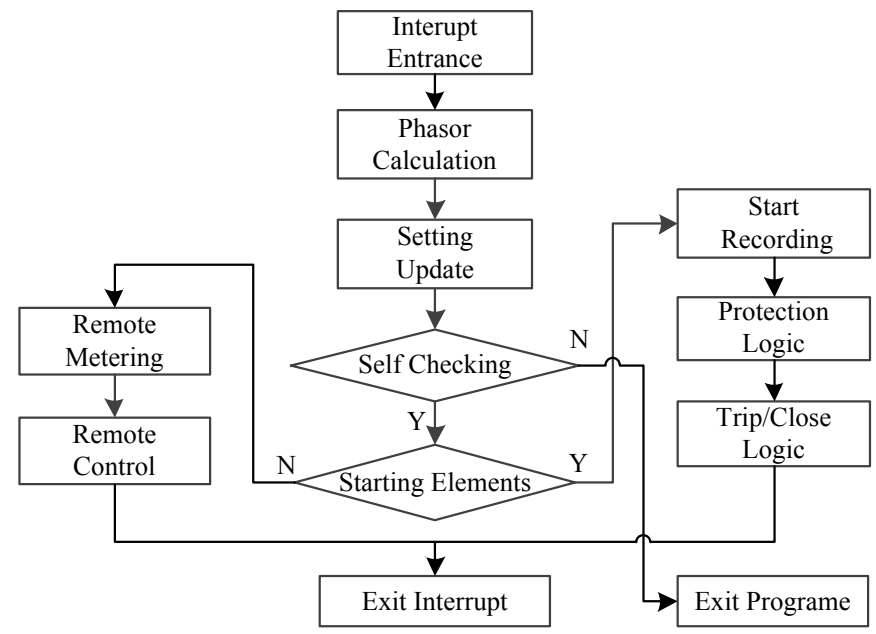

Fig.6 Flow chart of protection program

Phasor calculation program is used to calculate the phasors of electric analog quantities. Difference Fourier algorithm is adopted in the program. The real part and the imaginary part can be calculated by the following formula:

$$
\left\{\begin{array}{l}
X_{\mathrm{Re}}=\frac{2}{N K}\left(\frac{1}{2} \Delta X_{0}+\frac{1}{2} \Delta X_{\mathrm{N}}+\sum_{k=1}^{N-1} \Delta X_{\mathrm{k}} \cos n k \frac{2 \pi}{N}\right) \\
X_{\mathrm{Im}}=-\frac{2}{N K}\left(\sum_{k=1}^{N-1} \Delta X_{\mathrm{k}} \sin n k \frac{2 \pi}{N}\right)
\end{array}\right.
$$

where $\Delta X_{\mathrm{k}}=X_{\mathrm{k}}-X_{\mathrm{k}-1}, \mathrm{~N}$ is the number of sampling points which is related to SV Services in communication program, $K$ is difference coefficient and equals to $2 \sin \left(\frac{\pi}{N}\right)$.

Self-checking program checks the legitimacy of data such as three-phase currents and voltages, setting values and other configuration data. The protection program will send the alarm signal and then exit when checking fails. Starting elements include current mutation starting, current effective value starting, zero sequence current mutation starting, zero sequence current effective value starting and negative sequence current starting. If the starting elements act, the protection program starts to record fault waves and execute protection discrimination, else remote metering and remote control will be handled.

\section{Communication program}

The main task of the communication program is to process data for IEC61850 Ethernet communication. The program is designed based on TI-RTOS operating system, to simplify the program design. The IPC component of TI-RTOS provides efficient inter process communication between cores. FatFS (Fat File System) drivers perform data block transfers to and from physical media (NAND Flash and U Disk) as files. Since it is time-consuming for writing to and reading from physical media, based on the FatFS driver, higher-priority tasks can interrupt the file operation.

According to regulations, the OSI (Open Systems Interconnection) model is applied for IEC61850 Ethernet communication. The OSI model define seven layers, including Application layer, Presentation layer, Session layer, Transport layer, Network layer, Data link layer and Physical layer. In communication program, MMS Service corresponding to substation level, runs on Application layer of OSI model. Besides, in order to ensure the real-time ability, Services of process level communication, including SV Service, GOOSE Service and IEC1588 Service, are directly mapped to Data link layer after encoding. 
Based on Sockets APIs of TI-RTOS and MMS-Lite program package, we design the Application-layer services. The services of Data link layer are designed with EMAC (Ethernet Media Access Control) Drivers which directly operate hardwires.

Since Gigabit Ethernet is used in process layer communication, according to the existing research, packages of SV GOOSE and 1588 can be transmitted in one network ${ }^{[13]}$. In this case, IEC1588 Service has the highest priority, followed by SV Service, and then GOOSE Service. Thus, we divide the communication program into three processes:

1) Real-time process, including IEC1588 Service and SV Service which processing network packets by responding to Ethernet interrupts.

2) Quasi real-time process for GOOSE Service which processing GOOSE packets by responding a $0.5 \mathrm{~ms}$ timer interrupt.

3) Non real-time process, including MMS Service and file operation. This process runs in circular query way.

\section{Monitoring program}

Monitoring program is designed by GUI (Graphical User Interface) Composer of TI-RTOS. Device maximum supports $1024 \times 768$ LCD screen and can be operated by USB mouse and keyboard. In order to facilitate the operation, several drop-down menus have been designed in monitoring program. The structure of menus is shown in Fig.7.

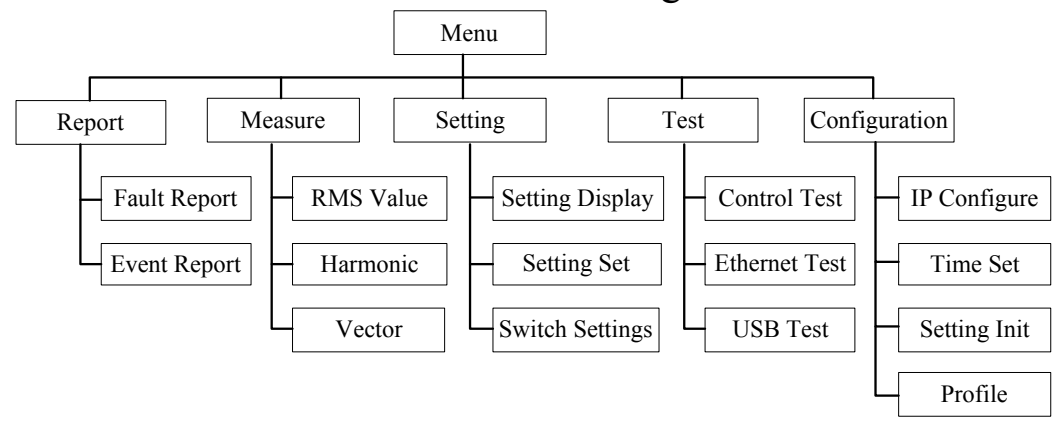

Fig.7 Structure of menus

As Fig.7, there are seven primary menus in the monitoring program, and each primary menu contains several secondary menus. The functions of protection, measurement and control can be realized through the menu operation.

\section{Conclusion}

In order to promote the application of digital relay protection in distribution network, the single-board micro digital relay protection device is introduced in this paper. The hardware platform of the device is composed of AM5728 embedded multicore processor, and it has advantages of high reliability, powerful computational capability and small size. Besides, due to the application of the advanced design tools of TI-RTOS, not only the software has powerful functions and optimized program structure, but also can greatly save the costs of development and maintenance. There for the micro digital relay protection device meets the general requirements of protection and monitor devices for digital substation in distribution network.

\section{Acknowledgement}

In this paper, the work is supported by National Natural Science Foundation of China (Project No.51477057)

\section{References}

[1] LI Jiuhu, Zheng Yuping, GU Shi Dong. Application of electronic instrument transformer in digital substation [J]. Automation of Electric Power Systems. 2007, 31(7):94-98.

[2] IEC 61850 Communication networks and system insubstations [M]. 2005. 
[3] Hui Hou, Tianqi Xu, Dahai You, Xianggen Yin, Wei Chen, Bo Wang, Xiongkai He. Research on Protection Equipment with Digital Interface Based on IEC-61850 [C]. Power Engineering Society General Meeting, 2007. IEEE , vol., no., pp.1-5, 24-28 June 2007

[4] Kai Ma, Shu Huang, Jiongcong Chen, Yingan Yang. Discussion of the testing technology for protection device used in digital substation [C]. Electricity Distribution (CICED), 2010 China International Conference on , vol., no., pp.1-5, 13-16 Sept. 2010

[5] Dong Xinzhou, Ding Lei. Research on design of digital integrated protection and control system [J]. Power System Protection and Control, 2009, 01:1-5.

[6] HAN Wei, YANG Xiaoming, QIU Xinhong, XING Yibao. Integrated Protection Device Based on Digital Sampling Technology [J]. Automation of Electric Power Systems, 2010,11:101-104.

[7] WANG Haiyan, XU Yunyan, WANG Shiyun, CUI Zhiguo. Digital Protection Based on DSP\&MPC8247 [J]. Automation of Electric Power Systems, 2010,09:112-115.

[8] Ning Yang, Wanjian Zhao, Yaoliang Xu, Shaocheng Zhang, Yi Zhu. Applications of DSP\&ARM for microprocessor protection device in distance protection. Computer Research and Development (ICCRD), 2011 3rd International Conference, vol.2, no., pp.227-231, 11-13 March 2011

[9] Gupta P., Khandelwal M.. Sharma R., Singh M.K., Reddy, N.P., Differential protection of transformer using FPGA. Smart Electric Grid (ISEG), 2014 International Conference on , vol., no., pp.1-6, 19-20 Sept. 2014

[10] Valsan S.P., Swarup K.S., Protective relaying for power transformers using field programmable gate array. in Electric Power Applications, IET , vol.2, no.2, pp.135-143, March 2008

[11]Jianguo Ruan; Jiajun Lin, "The application of DSP technique in the field of relay protection," in Communications, Circuits and Systems, 2005. Proceedings. 2005 International Conference on, vol.2, no., pp.1329, 27-30 May 2005

[12]Lin Wang, Chen F., Allen G., Cheung H., Mander T., Cheung R. Network-Integrated DSP-based Adaptive High Impedance Ground Fault Feeder Protection. in Power Engineering Society General Meeting, 2007. IEEE , vol., no., pp.1-7, 24-28 June 2007

[13]LIU Haoyu, FU Mingjun, ZUO Qunye, ZHANG Bao-shan. Research on sampling and network scheme of centralized protection based on tri-networks integration in process layer. Power System Protection and Control ,2013,21:141-146 